\title{
Reconstruction of the New Agegraphic Polytropic Gas Dark Energy Model in the Flat Friedmann Robertson Walker (FRW) Universe
}

\author{
P. Das*, K. P. Singh \\ Department of Mathematical Sciences, Bodoland University, Kokrajhar-783370, BTC, Assam, India
}

Received 11 January 2021, accepted in final revised form 3 June 2021

\begin{abstract}
In this paper, we study the Polytropic Gas Dark Energy model and New Agegraphic Dark Energy model in the flat Friedmann Robertson Walker (FRW) Universe and establish a correspondence between them for the scalar fields. This correspondence allows reconstructing the potential of the Polytropic Gas scalar fields and dynamics of the scalar fields according to the evolutions of the New Agegraphic Dark Energy, which describes the accelerated expansion of the Universe.
\end{abstract}

Keywords: Accelerated expansion; New agegraphic dark energy; Polytropic gas.

() 2021 JSR Publications. ISSN: 2070-0237 (Print); 2070-0245 (Online). All rights reserved. doi: http://dx.doi.org/10.3329/jsr.v13i3.51323

J. Sci. Res. 13 (3), 779-784 (2021)

\section{Introduction}

A number of cosmological experiments and observational data confirm that our Universe is expanding with acceleration, and new energy with negative pressure, called dark energy (DE) is responsible for this expansion [1-9]. The nature of the DE is still unknown though a number of candidates have been proposed by the researchers in this field. About $70 \%$ of the present energy of the Universe is considered DE. The cosmological constant is the earliest and simplest candidate for dark energy. But the cosmological constant has two difficulties, namely "fine-tuning" and "cosmic coincidence" problems [10,11]. For that reason, many alternative dark energy models such as the quintessence $[12,13]$, phantom [14-16], K-essence [17-19], tachyon [20-22], dilaton [23-25], etc. have been proposed by the researchers. But all of these models are not highly acceptable. Some dark energy models are based on fundamental principles and laws. The Agegraphic models are such kind of dark energy models. The Agegraphic dark energy model is based on the Karolyhazy relation [26]

$$
\delta t=\beta t_{P}^{\frac{2}{3}} t^{\frac{1}{3}}
$$

Where $\beta$ is a dimensionless constant and $t_{P}=l_{P}=\frac{1}{M_{P}}$ with $t_{P}, l_{P}$ and $M_{P}$ being the reduced Planck time, length and mass, respectively. There are two types of Agegraphic

\footnotetext{
*Corresponding author: prasantadasp4@gmail.com
} 
models: Original Agegraphic Dark Energy (OADE) and New Agegraphic Dark Energy (NADE) models. The OADE model is proposed by R. G. Cai to explain the accelerated expansion of the Universe based on the uncertainty relation of quantum mechanics and the gravitational effect in general relativity. But the OADE model has some difficulties. Because it cannot justify the matter-dominated era [27]. This motivated H. Wei and R. G. Cai [28] to propose the NADE model, where the time scale is chosen to be the conformal time instead of the age of the Universe.

In this paper, we will focus on the New Agegraphic Dark Energy model and Polytropic Dark Energy models in the flat FRW Universe. Since the present Universe is homogeneous and isotropic as per the cosmological principle and the Friedmann Robertson Walker (FRW) metric satisfies the cosmological principle, so our Universe is FRW Universe. Also, many cosmologists indicate that the present Universe will expand forever, but the rate of expansion gradually tends to zero after an infinite amount of time. This is the condition for a zero curvature Universe or flat Universe. Moreover, Ahmed and Alamri found that the flat FRW Universe is stable [29]. Therefore, our model is considered for the flat FRW Universe. This paper is organized as follows. In section 2, we study the Polytropic Gas Dark Energy model. In section 3, we investigate the NADE in the flat FRW Universe. In section 4, we establish a correspondence between the NADE and the Polytropic Gas model of DE and reconstruct the potential and the dynamics for the scalar field of the Polytropic model, which describe an accelerated expansion Universe. Finally, in section 5, conclusions are given.

\section{Polytropic Gas Dark Energy Model}

The equation of state (EOS) of the Polytropic Gas [30,31] is given by

$$
P_{\Lambda}=K \rho_{\Lambda}^{1+\frac{1}{n}}
$$

Where $P_{\Lambda}, \rho_{\Lambda}, K$ and $n$ are the pressure, energy density, Polytropic constant, and Polytropic index, respectively.

The conservation equation for the dark energy in the FRW Universe is given by

$$
\dot{\rho}_{\Lambda}+3 H\left(\rho_{\Lambda}+P_{\Lambda}\right)=0
$$

Where $H$ the Hubble parameter and overhead dot is denoting the differentiation with respect to the cosmological time

Using the EOS (2) into the conservation eq. (3) and integrating we get

$$
\rho_{\Lambda}=\left[B a^{3 / n}-K\right]^{-n}
$$

Where $B$ is a positive integration constant and $a(t)$ is a time scale factor of the Universe. The corresponding pressure takes the following form

$$
P_{\Lambda}=K\left[B a^{3 / n}-K\right]^{-n-1}
$$

Using eqs. (3) and (4), the EOS parameter for the Polytropic Gas Dark Energy model is obtained as 


$$
\omega_{\Lambda}=\frac{P_{\Lambda}}{\rho_{\Lambda}}=-1+\frac{B a^{3} / n}{B a^{3 / n-K}}
$$

When $K>B a^{3 / n}$, from (5), we see that $\omega_{\Lambda}<-1$, which corresponds to a Universe dominated by phantom field. The phantom field lead to the accelerated expansion of the Universe

The pressure and energy density for the scalar field $\varphi$ are given by

$$
\begin{aligned}
& P_{\varphi}=\frac{1}{2} \dot{\varphi}^{2}-V(\varphi) \\
& \rho_{\varphi}=\frac{1}{2} \dot{\varphi}^{2}+V(\varphi)
\end{aligned}
$$

From the eqs. (3), (4), (7) and (8), we get the scalar potential and the kinetic energy terms for the Polytropic Gas as

$$
\begin{aligned}
& V(\varphi)=\frac{\frac{B}{2} a^{3 / n-K}}{\left(B a^{3 / n-K}\right)^{n+1}} \\
& \dot{\varphi}^{2}=\frac{B a^{3 / n}}{\left(B a^{3 / n-K}\right)^{n+1}}
\end{aligned}
$$

When $K>B a^{3 / n}$, then from (10), we see that $\dot{\varphi}^{2}<0$ and it indicates that the scalar field $\varphi$ is a phantom field.

\section{New Agegraphic Dark Energy Model in the Flat FRW Universe}

The energy density of the New Agegraphic Dark Energy for a flat Universe is given by [32]

$$
\rho_{\Lambda}=\frac{3 n^{2} M_{P}^{2}}{\eta^{2}}
$$

Here $M_{P}=(8 \pi G)^{-\frac{1}{2}}, n$ is a numerical parameter and $\eta$ is considered as the conformal age of the Universe defined by

$$
\eta=\int \frac{d t}{a}=\int \frac{d a}{H a^{2}}
$$

Here $a$ is the scale factor of the Universe, $H=\frac{\dot{a}}{a}$ is the Hubble parameter and the dot denotes the derivatives with respect to cosmic time.

The corresponding fractional energy density is

$\Omega_{\Lambda}=\frac{\rho_{\Lambda}}{\rho_{c r}}$, where $\rho_{c r}=3 M_{P}^{2} H^{2}$ is the critical energy density of the Universe. So, from the eq. (11), we have

$$
\begin{aligned}
& \rho_{\Lambda}=3 M_{P}^{2} H^{2} \Omega_{\Lambda} \\
& \Omega_{\Lambda}=\frac{n^{2}}{\eta^{2} H^{2}}
\end{aligned}
$$

and

$$
\eta=\frac{n}{H \sqrt{\Omega_{\Lambda}}}
$$


It gives the conformal time of the Universe in terms of the fractional energy density of dark energy.

Differentiating (11) with respect to cosmic time and using $\dot{\eta}=\frac{1}{a}$, in (15) we get

$$
\dot{\rho_{\Lambda}}=-\frac{2 H \sqrt{\Omega_{\Lambda}}}{n a} \rho_{\Lambda}
$$

From the eq. (16) and (3), we can find the equation of state (EoS) parameter of the New Agegraphic Dark Energy as follows

$$
\omega_{\Lambda}=-1+\frac{2}{3 n} \frac{\sqrt{\Omega_{\Lambda}}}{a}
$$

When $a \rightarrow \infty$ and $\Omega_{\Lambda} \rightarrow 1$ then $\omega_{\Lambda} \rightarrow-1$ in the late time. At present, i.e., if $a=1$, $\Omega_{\Lambda}=1$ then $\omega_{\Lambda}<-1$ for $n<0$ and $\omega_{\Lambda}>-1$ for $n>0$

\section{Correspondence between the Polytropic Gas and New Agegraphic Dark Energy Models}

To establish the correspondence between the Polytropic Gas and New Agegraphic Dark Energy models for the scalar fields, we compare the New Agegraphic Dark Energy density (13) with the Polytropic Gas model density (4) and equate the EoS parameter of the New Agegraphic Dark Energy (17) with the EoS parameter of the Polytropic Gas model (6).

Now comparing the eq. (4) and (13), we get

$$
3 M_{P}^{2} H^{2} \Omega_{\Lambda}=\left[B a^{3 / n}-K\right]^{-n}
$$

and

$$
K=B a^{\frac{3}{n}}-\left(3 M_{P}^{2} H^{2} \Omega_{\Lambda}\right)^{-\frac{1}{n}}
$$

Again from the eqs. (6), (17) and (19)

$$
K=\left(3 M_{P}^{2} H^{2} \Omega_{\Lambda}\right)^{-\frac{1}{n}}\left(-1+\frac{2 \sqrt{\Omega_{\Lambda}}}{3 n a}\right)
$$

Using (20) in (19), we get

$$
B=\left(3 M_{P}^{2} H^{2} \Omega_{\Lambda} a^{3}\right)^{-\frac{1}{n}}\left(\frac{2 \sqrt{\Omega_{\Lambda}}}{3 n a}\right)
$$

Now we reconstruct the scalar potential (9) and the kinetic energy term (10) using the equations (20) and (21) in the following forms

$$
\dot{\varphi}^{2}=\left(2 M_{P}^{2} H^{2}\right)\left(\frac{\left(\Omega_{\Lambda}\right)^{\frac{3}{2}}}{n a}\right)
$$

and

$$
V(\varphi)=3 M_{P}^{2} H^{2} \Omega_{\Lambda}\left(1-\frac{2 \sqrt{\Omega_{\Lambda}}}{3 n a}\right)
$$

The equation (23) is the scalar potential of the Polytropic Gas Dark Energy according to the evolution of the New Agegraphic Dark Energy. 
We can rewrite the eq. (22), using definition $\dot{\varphi}=\varphi^{/} H$ and changing the time derivative to the derivative with respect to $x=\ln a$ in the following form

$$
\varphi^{\prime}=M_{P}\left(\frac{2\left(\Omega_{\Lambda}\right)^{\frac{3}{2}}}{n a}\right)^{\frac{1}{2}}
$$

Finally, the evolutionary form of a scalar field is obtained as

$$
\varphi(a)-\varphi(0)=\int_{0}^{\ln a} M_{P}\left(\frac{2\left(\Omega_{\Lambda}\right)^{\frac{3}{2}}}{n a}\right)^{\frac{1}{2}} d x
$$

For the present time, we take $a_{0}=1$. Thus, the corresponding scalar field of the Polytropic Gas Dark Energy model has been reconstructed based on the evolution of the New Agegraphic Dark Energy model. This reconstruction indicates that the Universe expands with acceleration.

\section{Conclusion}

In this work, we consider the Polytropic Gas Dark Energy model and New Agegraphic Dark Energy model for the flat FRW Universe. For $K>B a^{3 / n}$, the Polytropic Gas model behaves as a phantom-like dark energy model, indicating that the Universe expands with acceleration. The New Agegraphic Dark Energy model also almost behaves like a cosmological constant when $a \rightarrow \infty$ and dark energy dominated. We have proposed a correspondence between the Polytropic Gas Dark Energy model and New Agegraphic Dark Energy model for the scalar fields. We reconstruct the potential of the Polytropic Gas scalar fields and dynamics of the scalar fields according to the evolutions of the New Agegraphic Dark Energy, which describes the accelerated expansion of the Universe.

\section{References}

1. A. G. Riess, et al., Astron. J. 116, 1009 (1998).

2. S. Perlmutter, et al., Astrophys. J. 517, 565 (1999).

3. P. De Bernardis, et al., Nature, 404, 955 (2000). https://doi.org/10.1038/35010035

4. D. N. Spergel, et al., Astrophys. J. Supplement Series 148, 135 (2003). https://doi.org/10.1086/377226

5. R. A. Knop, et al., Astrophys. J. 598, 102 (2003).

6. R. R. Caldwell and M. Doran, Phys. Rev. D 69, ID 103517 (2004). https://doi.org/10.1103/PhysRevD.69.103517

7. M. Tegmark, et al., Phys. Rev. D 69, ID 103501 (2004). https://doi.org/10.1103/PhysRevD.69.103501

8. T. Koivisto and D. F. Mota, Phys. Rev. D 73, ID 083502, 2006. https://doi.org/10.1103/PhysRevD.73.083502

9. S. F. Daniel, R. R. Caldwell, A. Cooray, and A. Melchiorri, Phys. Rev. D, 77, ID 103513 (2008). https://doi.org/10.1103/PhysRevD.77.103513

10. S. Weinberg, Rev. Mod. Phys. 61, 1 (1989). https://doi.org/10.1103/RevModPhys.61.1

11. S. M. Carroll, Liv. Rev. Relativ. 1, 1 (2001).

12. C. Wetterich, Nucl. Phys. B 302, 668 (1988). https://doi.org/10.1016/0550-3213(88)90193-9 


\section{New Agegraphic Polytropic Gas Dark Energy Model}

13. B. Ratra and P. J. E. Peebles, Phys. Rev. D 37, 321, 1988. https://doi.org/10.1103/PhysRevD.37.3406

14. R. R. Caldwell, Phys. Lett. B 545, 23 (2002). https://doi.org/10.1016/S0370-2693(02)02589-3

15. S. Nojiri and S. D. Odintsov, Phys. Lett. B 562, 147 (2003). https://doi.org/10.1016/S0370-2693(03)00546-X

16. S. Nojiri and S. D. Odintsov, Phys. Lett. B 565, 1 (2003). https://doi.org/10.1016/j.physletb.2003.08.013

17. T. Chiba, T. Okabe, and M. Yamaguchi, Phys. Rev. D 62, ID 023511 (2000). https://doi.org/10.1103/PhysRevD.62.023511

18. C. Armendariz-Picon, V. Mukhanov, and P. J. Steinhardt, Phys. Rev. Lett. 85, 4438 (2000). https://doi.org/10.1103/PhysRevLett.85.4438

19. C. Armendariz-Picon and V. Mukhanov, Phys. Rev. D 63, ID 103510 (2001). https://doi.org/10.1103/PhysRevD.63.103510

20. T. Padmanabhan, Phys. Rev. D 66, ID 021301 (2002). https://doi.org/10.1103/PhysRevD.66.021301

21. T. Padmanabhan and T. R. Choudhury, Phys. Rev. D 66, ID 081301 (2002). https://doi.org/10.1103/PhysRevB.66.081301

22. A. Sen, J. High Energy Phys. 04, ID 048 (2002). https://doi.org/10.1088/11266708/2002/04/048

23. M. Gasperini, F. Piazza, and G. Veneziano, Phys. Rev. D 65, ID 023508 (2002). https://doi.org/10.1103/PhysRevD.65.023508

24. F. Piazza and S. Tsujikawa, J. Cosmol. Astropart. Phys. 07, ID 004 (2004). https://doi.org/10.1088/1475-7516/2004/07/004

25. N. Arkani-Hamed, P. Creminelli, S. Mukohyama, and M. Zaldarriaga, J. Cosmol. Astropart. Phys. 04, 001 (2004). https://doi.org/10.1088/1475-7516/2004/04/001

26. F. Karolyhazy, Nuovo Cimento A 42, 390 (1966). https://doi.org/10.1007/BF02717926

27. R. G. Cai, Phys. Lett. B 657, 228 (2007). https://doi.org/10.1016/j.physletb.2007.09.061

28. H. Wei and R. G. Cai, Phys. Lett. B, 660, 113 (2008). https://doi.org/10.1016/j.physletb.2007.12.030

29. N. Ahmed and S. Z. Alamri, Int. J. Geom. Methods Mod. Phys. 16, ID 1950159 (2019). https://doi.org/10.1142/S0219887819501597

30. J. Christensen-Dalsgard, Lecture Notes on Stellar and Structure and Evolution, $6^{\text {th }}$ Edition (Aarhus University Press, Aarhus, 2004).

31. P. Das and K. P. Singh, Int. J. Adv. Res. Eng. Technol. 11, 241 (2020).

32. A. Sheykhi, Phys. Lett. B 680, 113 (2009). https://doi.org/10.1016/j.physletb.2009.08.051 